\title{
Need for Clinical Trial Registry and Institutional Review Board Approval in Human Subjects Research

\author{
Soo Young Kim
}

Department of Family Medicine, Kangdong Sacred Heart Hospital, Hallym University College of Medicine, Seoul, Korea

In 2013, two important changes will occur in the Korean Journal of Family Medicine (KJFM) policy. One is mandatory registration of clinical trials before submission to KJFM, and the other is to required institutional review board (IRB) approval of all research involving human subjects.

A clinical trials registry is an official platform for registering a clinical trial. ClinicalTrials.gov, run by the US National Library of Medicine, was the first online registry for clinical trials and is the largest and most widely used today. The goal of a clinical trials registry is to provide increased transparency and access to clinical trials to the public. ${ }^{1)}$ Clinical trials registries may decrease selective outcome reporting and further selective reporting bias. ${ }^{2)}$

The International Committee of Medical Journal Editors decided that from July 1, 2005 no trials will be considered for publication unless they are included on a clinical trials registry. The World Health Organization has begun the push for clinical trial registration with the initiation of the International Clinical Trials Registry Platform. The Clinical Research Information Service is South Korea's registry, and is available http://cris. nih.go.kr/cris/index.jsp. It is managed by the Korea Centers for Disease Control and Prevention and funded by South Korea's Ministry of Health and Welfare. ${ }^{4)}$

Guidelines for authors of many journals may also contain such requirements. For example, in instructions for authors of the Journal of the American Medical Association, we can find such a policy as follows. "All clinical trials must be registered at an appropriate online public registry." However, the KJFM does not have such a policy for its authors.

In Korea, the Bioethics and Safety Act was enacted in 2008 and was revised to reflect the rapidly changing trends in the field. It will be effective from February 2, 2013.

An IRB is a committee that has been formally designated to approve, monitor, and review biomedical and behavioral research involving humans. By the revised act, all research involving human subjects must be approved by the IRB. In the information for authors of the KJFM, it is stated, "If the research involves human life, it must comply with the ethical standards of the 1975 Helsinki
Declaration (revised edition of 2004) and in principle undergo scrutiny of an independent IRB which reviews ethical issues of the human experiment. But, in clinical studies, the approval of the IRB and patient's consent must be received and stated on paper." We must change our guidelines according to the revised act by changing 'clinical studies' to "human subjects research."

Some researchers may think that the need for a clinical trial registry and IRB approval in human subjects research are obstacles in the research process; however, adhering to such guidelines will bring us closer to our goal of becoming a leading journal in the international medical community.

\section{CONFLICT OF INTEREST}

No potential conflict of interest relevant to this article was reported.

\section{REFERENCES}

1. Wikipedia. Clinical trials registry [Internet]. St. Petersburg: Wikipedia [cited 2012 Jul 11]. Available from: http:// en.wikipedia.org/wiki/Clinical_trials_registry.

2. International Committee of Medical Journal of Editors. Uniform requirements for manuscripts submitted to biomedical journals: publishing and editorial issues related to publication in biomedical journals: obligation to register clinical trials [Internet]. Philadelphia: International Committee of Medical Journal of Editors; c2009 [cited 2013 Jan 3]. Available from: http://www.icmje.org/ publishing_10register.html.

3. Kim SY. Selective reporting and clinical trial registry. Korean J Fam Med 2009;30:923.

4. Kim SE. Registration and reporting guidelines for clinical trials. Korean J Med 2012;83:309-18. 